\title{
Update on Allergy Immunotherapy
}

\author{
William Davidson, MD; Sean Lucas, MD; Larry Borish, MD
}

\begin{abstract}
This article summarizes and provides commentary regarding guidelines on the administration of immunotherapy (IT) for allergic airway disease. Recent investigations have provided important insights into the immunologic mechanism of IT and the prominent role of interleukin-10-producing regulatory $T$ lymphocytes. The most important aspect of successful IT is the administration of an appropriate dose of an extract containing a sufficient concentration of the relevant allergen. This is largely possible now only with standardized extracts. When the major allergen content of successful IT extracts was quantified, efficacy was demonstrated across a surprisingly narrow concentration range (approximately 5-24 $\mu \mathrm{g}$ per injection), irrespective of the extract. This presumably reflects the concentration of an antigen that drives an immune response toward tolerance. It may be predicted that as major allergen content is quantified in currently nonstandardized extracts, effective IT will also be achieved by administering a dose in this range, in contrast to current practices involving fairly arbitrary dosing decisions. With the availability of nonsedating antihistamines, intranasal corticosteroids, and the leukotriene modifiers, inadequate pharmacologic response or intolerable side effects are less commonly the major indications for starting IT for allergic rhinitis (AR). However, with the recognition that a relatively short course (3-5 years) of IT can provide long-term immunomodulation and clinical benefit, a desire to avoid longterm pharmacotherapy and the associated high costs may be the primary indication for IT in AR cases. While evidence overwhelmingly supports the beneficial influences of IT in asthma cases, the positioning of IT for this disorder is not established. The observed prevention of asthma in children who have AR is intriguing, but further studies are required to assess the extent to which the prevalence and severity of chronic asthma will be reduced when these children reach adulthood. Similarly, safety issues overwhelmingly suggest that uncontrolled asthma is the greatest risk factor for mortality associated with IT and that IT therefore may be contraindicated for most patients who have inadequate pharmacologic responses or are unable to tolerate useful pharmacologic agents. Paradoxically, these are the patients for whom a response to IT may be most desirable.
\end{abstract}

This article summarizes and provides commentary regarding current guidelines on the administration of immunotherapy (IT) for allergic airway disease. Details on currently accepted practices in the preparation and administration of IT are published

Asthma and Allergic Disease Center, University of Virginia Health Systems, MR4 Bldg., Rm. 5041, Lane Rd., Charlottesville, Virginia, USA 22908

Correspondence to: Dr. Larry Borish, MD, Asthma and Allergic Disease Center, PO Box 801355, University of Virginia Health Systems, Charlottesville, Virginia, USA 22908-1355. E-mail: 1b4m@virginia.edu

Supported by: NIH: PO1 AI50989 and RO1 AI47737. elsewhere, ${ }^{1-5}$ and allergy practitioners are advised to use these references as the standard of care. This article draws primarily on recent studies regarding the current understanding of the mechanism and proper administration of IT (with emphasis on the importance of properly dosing major allergen content) and on current insights into the selection of patients and allergens for IT. Finally, several recent studies have reported on issues of morbidity and mortality associated with IT and emphasize particularly the importance of postponing administration of IT at times when asthma is poorly controlled. 


\section{Mechanisms of Immunotherapy}

Several mechanisms of IT have emerged over recent decades, reflecting an increased understanding of immunology. Early studies concentrated on increases in allergen-specific immunoglobulin ( $\mathrm{Ig}$ ) $\mathrm{G}$, specifically that of the IgG4 isotype. These immunoglobulins were proposed as "blocking" immunoglobulins that competed with IgE for allergen binding to IgE receptor-expressing cells. However, the correlation between IgG concentrations and clinical response to treatment is weak, and even when correlated to nasal $\operatorname{IgG}$ (or $\operatorname{Ig} \mathrm{A}$ ) concentrations in the allergic inflammatory milieu, no improvements in these relationships were observed.

More recently, immunodeviation has been cited, with reductions in $\mathrm{T}$-cell proliferative responses to allergens and a shift from a T-helper 2(Th2)-like response toward a Th1-like response following immunotherapy. ${ }^{6}$ Unfortunately, this model also comes under scrutiny because (1) T cells that produce interferon (INF)- $\gamma$ (Th1-like cells) are a characteristic feature of allergic inflammation, (2) IFN- $\gamma$ is a potent proinflammatory compound that contributes to both the presence and severity of allergic disease, ${ }^{7}$ and (3) subsequent studies have failed to consistently confirm these findings. In contrast, the one consistent finding observed after IT is diminished responsiveness (tolerance) of the allergen-specific Th2-like cells.

As such, a potentially more valid model attributes the mechanism of IT to the induction of T cells with regulatory activity. Several classes of regulatory $\mathrm{T}$ cells have been described (Table 1), including IL-10-producing lymphocytes (termed "Tr1 cells"), CD $25^{+} \mathrm{T}$ regulatory (Treg) cells, and Th3 cells that produce transforming growth factor beta (with or without IL-10). Thymus-derived CD25 $5^{+}$ Treg cells are important for the induction of tolerance to self-antigens and the prevention of autoimmunity. Th 3 cells are primarily ascribed to mucosal tolerance. Whereas these latter two cell groups are therefore unlikely to be involved in allergen IT, induction of Tr1 cells may play a key role in reducing allergen-specific T-cell responsiveness.

A prominent role for IL-10-producing activated $\mathrm{CD}^{+}$cells was first described in studies involving bee venom IT. ${ }^{8}$ Subsequent investigations with house dust mite IT extended the importance of IL-10 (and transforming growth factor beta [TGF- $\beta$ ]) production by $\mathrm{CD} 4^{+} \mathrm{T}$ cells to inhalant allergy and confirmed that this occurred in parallel to the suppression of Th2 proliferative responses and cytokine production. ${ }^{9}$ In this study, IL-10 responses in healthy nonatopic individuals who had been exposed to allergen were similar to those in the IT-treated group, implying the restoration of tolerant $\mathrm{T}$-cell responses in the atopic

Table $1 \mathrm{CD4}^{+} \mathrm{T}$ Cells with Regulatory Activity

\begin{tabular}{ll}
\hline Regulatory T Cell & Characteristics \\
\hline Treg & CD25+ foxp3+ thymus derived \\
& Not dependent on IL-10 for biologic activity \\
& Mediates self-tolerance; prevents autoimmune disease \\
& Not likely to be relevant to acquired tolerance to allergens \\
& Characterized by TGF- $\beta$ ( \pm IL-10) production \\
Th3 & Mediates mucosal tolerance/antigen-specific IgA production \\
& Not relevant to allergy or immunotherapy \\
& Peripheral-derived regulatory T cells \\
Tr1 & IL-10 responsible for biologic activity $( \pm$ TGF- $\beta$ ) \\
& Possibly derived from Th1- or Th2-like lymphocytes or natural T cells \\
& CD25 expression (reflecting activation) \\
& Foxp3 negative \\
& Proposed mechanism of immunotherapy \\
\end{tabular}

IgA = immunoglobulin A; IL-10 = interleukin-10; TGF- $\beta=$ transforming growth factor beta; $\mathrm{Th}=\mathrm{T}$ helper; $\operatorname{Tr} 1=$ peripherally-derived regulatory $\mathrm{T}$ cell; Treg $=$ thymic-derived regulatory $\mathrm{T}$ cells . 
Table 2 Clinical Indications for Allergen Immunotherapy

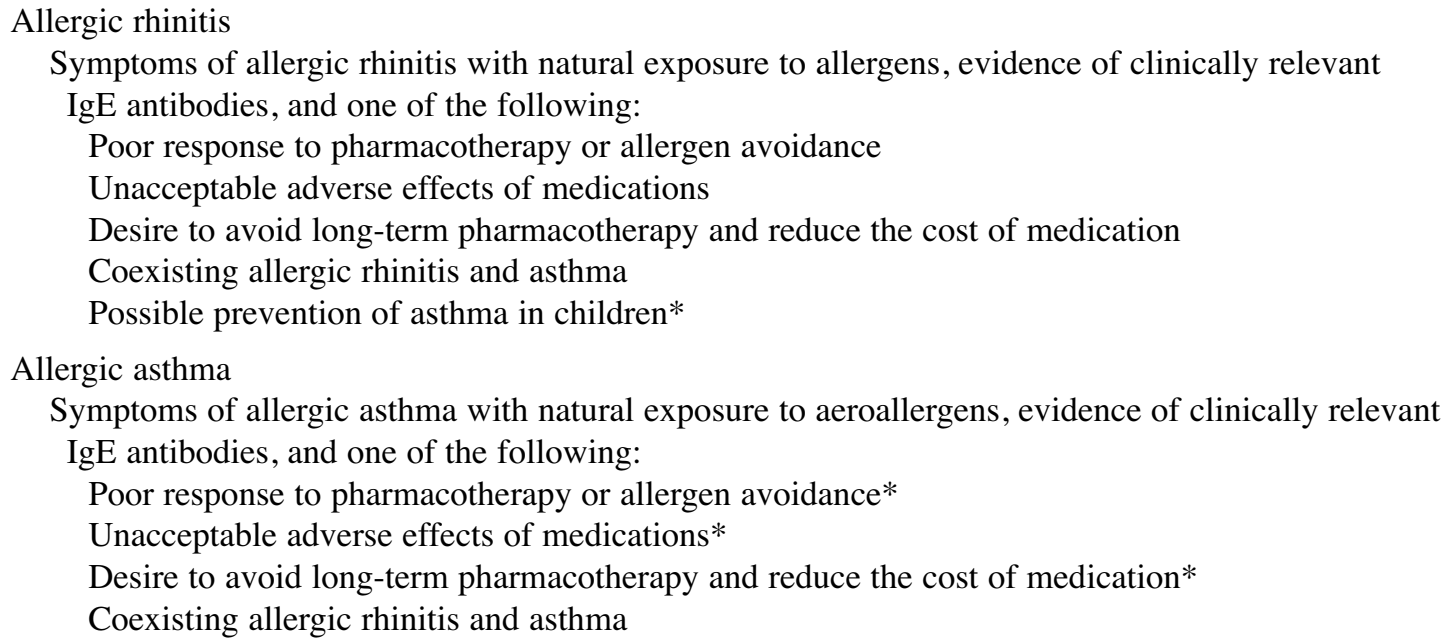

individual. Other studies have also shown IL-10 production by $\mathrm{CD}^{+}$cells without changes in grass pollen-induced proliferation or Th2 cytokine production..$^{10}$ This area of research remains confused; for example, although a role for CD25 expression has been ascribed to these IL-10-producing cells, it is unclear whether this reflects the constitutive expression of this component of the IL-2 receptor that is the signature characteristic of Treg cells or whether this reflects the induced expression of this component of the IL-2 receptor as occurs with activation of these effector T cells. However, what is consistent is that each of these studies has found cells capable of making high levels of IL-10 (with or without TGF- $\beta$ ) consistent with the Trl cell type, and current concepts therefore focus on the integral role of these IL-10-producing cells in immune tolerance to allergens after IT.

\section{Indications for Aeroallergen IT}

Although allergen IT is effective as a treatment of allergic rhinitis and allergic asthma, considerations reflecting its variable degree of therapeutic benefit and safety have led to the absence of consistent recommendations regarding indications for allergen IT for these conditions. A task force representing the American Academy of Allergy, Asthma and Immunology and the American College of Allergy, Asthma \& Immunology recently published evidence-based guidelines regarding indications for IT for inhaled allergens (Table 2), ${ }^{5}$ and similar recommendations were previously published by the Canadian Society of Allergy and Clinical Immunology. ${ }^{1}$

\section{Allergic Rhinitis}

Less controversy surrounds the use of IT for allergic rhinitis (AR).$^{2-4,11-15}$ Clearly, an absolute prerequisite for initiating IT is documentation of symptomatic disease, with evidence of specific $\operatorname{IgE}$ antibodies as shown by skin testing or by $\operatorname{IgE}$ immunoassays. Historically, the most important indications for IT were the combination of either a poor response to pharmacotherapy or allergen avoidance and unacceptable adverse effects from available medications. Given all of the limitations of allergen avoidance and the frequently inadequate relief provided by antihistamines, IT was often the best treatment available during the era when the only available agents for AR were sedating antihistamines. The introduction of second-generation nonsedating antihistamines gen- 
erally eliminated intolerance to antihistamines as an indication for IT. In addition, intranasal corticosteroids were made available and were shown, in frequent contrast to antihistamines, to be extremely effective treatments of AR. Although associated with local side effects, intranasal corticosteroids are generally well tolerated. More recently, leukotriene modifiers have been studied and have been shown to have some efficacy in treating AR although their ultimate position in treatment has not been determined. Thus, most patients can achieve effective control of their AR with pharmacologic treatments, without unacceptable adverse effects. As such, in addition to patients' reluctance to use intranasal corticosteroids, the most important indication for initiating IT for AR is the patient's desire to avoid longterm pharmacotherapy and to reduce costs. This finding is primarily supported by a seminal study demonstrating that IT-induced relief from the symptoms of grass pollen rhinitis persisted for at least 3 years after the discontinuation of IT. ${ }^{16}$ Given supportive earlier studies suggesting that 3 to 5 years of IT can provide long-term benefits, ${ }^{17}$ the preference for short-term IT over life-long pharmacotherapy may be the most important indication for recommending IT. Although cures are unusual with IT, patients who are allergic to allergens for which effective agents are available can expect significant clinical benefit when treated at appropriate dosages (as discussed below). Thus, many of these patients can hope to evolve from being dependent on continuous multidrug therapy (including topical corticosteroids) to perhaps being manageable with as-needed antihistamines alone. Clearly, 3 to 5 years of IT has cost benefits when compared to lifelong pharmacotherapy. In addition, although IT requires a significant time commitment and is associated with a certain discomfort to the patient, many patients will accept this commitment in exchange for eliminating a lifelong dependence on multidrug therapy.

The role of IT in patients with coexisting asthma is discussed below. The indication that IT can prevent the development of asthma in children is intriguing but still controversial. ${ }^{18-20}$ This indication is based primarily on a multicentre study of children with AR that found that 3 years of IT for grass and/or birch pollen allergy could reduce the risk of asthma developing later. ${ }^{20}$ This recommendation may not be wholly appropriate for several reasons. Patients, particularly children, with severe seasonal allergic rhinitis (SAR) often have pulmonary complaints of cough and chest tightness. However, these patients generally have a fairly benign syndrome, and their lower airway complaints often respond to whatever therapies are offered for their SAR, including intranasal corticosteroids and antihistamines. This study provides compelling evidence that these patients do in fact have asthma. What this study does not establish is whether IT administered at this early stage as treatment of AR can prevent the later development of persistent (as opposed to "seasonal") asthma. Specifically, it is unknown whether a difference in prevalence and severity of asthma will persist after these patients reach adulthood. Arguably, this study merely added IT to intranasal corticosteroids and antihistamines as AR-directed therapies that might mitigate this benign form of "seasonal allergic asthma." The frequency with which asthma developed in this study far exceeded what would be expected regarding the development of asthma in an atopic cohort and may suggest that the investigators were reporting on the mitigation of a less severe asthma variant. A second study also reported that the risk of asthma symptoms was higher (threefold) in controls than in asthmatic subjects who received IT. ${ }^{19}$ However, this study did not demonstrate any differences in lung function between experimental and control groups, nor was there any significant difference between the two groups in regard to the use of asthma medications. Additional studies with longer-term follow-ups are still required to confirm whether IT administered in infancy can reduce the prevalence of persistent asthma later in life.

\section{Allergic Asthma}

The second indication for IT for patients with aeroallergen sensitization is for allergic asthma. Although the efficacy of IT for asthma is viewed as controversial and many studies provide conflicting evidence, the preponderance of data clearly 
supports a beneficial influence of IT in this disorder. ${ }^{15,21-25}$ Recent meta-analyses of approximately 75 asthma trials concluded that IT reduced the use of asthma rescue medications and the frequency of asthma symptoms..$^{26,27}$ These studies conceded, however, that the evidence gave limited guidance regarding the size of the benefit relative to that of other therapies. Because of this and because of safety concerns, the role of IT in allergic asthma remains controversial. For example, the first criterion listed in the guidelines, "poor response to pharmacotherapy or allergen avoidance," is debatable, given safety issues. A poor response to pharmacotherapy implies uncontrolled asthma, and, as discussed below, uncontrolled asthma is considered a virtually absolute contraindication to IT.

Although IT can improve asthma symptoms and the need for a rescue $\beta$-agonist, ${ }^{26}$ there have been no well-performed controlled trials that have confirmed the claim that the administration of IT will help "avoid long-term pharmacotherapy and reduce the cost of medication." "Specifically, what has never been validated is the reasonable expectation that IT might reduce a patient's symptom severity to such an extent that one or more longterm controllers could be eliminated. Because the greatest concern regarding adverse effects is for those of oral and (arguably) inhaled corticosteroids, it would be imperative to generate data showing the ability of IT to eliminate the need for this class of medication. For corticosteroid-dependent asthma cases, the second criterion, like the first, is problematic insofar as asthma this severe should be viewed as a virtually absolute contraindication to starting IT. This leaves coexisting AR and (wellcontrolled) asthma as the most important indications for IT for asthma. It is reasonable to administer IT to an AR patient whose asthma is well controlled, with the recognition that the AR is likely to substantially improve and that any subsequent benefit to the asthma is an added "bonus."

\section{Efficacy of Allergy Immunotherapy}

It is reasonable to speculate that since its inception, IT has been attempted with virtually all con- ceivable aeroallergens. However, efficacy has been established against only a relatively few extracts; for many extracts, especially mould extracts, the evidence for efficacy is sparse. In welldesigned placebo-controlled studies, efficacy has been shown against ragweed, various grasses (timothy, rye grass), trees (mountain cedar, birch), dust mites, and cats. As discussed, significant clinical improvement has been shown for subjective symptom and medication diary scores in comparison to the placebo group. Efficacy largely requires definition of the nature of the specific allergen and the development of a means of generating an extract with a concentration of the relevant allergenic component sufficient to produce an immunomodulating effect (dosing requirements are discussed below). With pollens, defining the relevant allergen - the pollen grain itself - and developing the botanic techniques to obtain that allergen are relatively straightforward. Thus, the earliest studies categorically establishing the efficacy of IT were performed with ragweed, and subsequent studies have shown positive results with northern grasses and a few trees. The ability of studies to show the efficacy of IT for ragweed was rendered easier by the relative paucity of allergens responsible for autumnal seasonal AR and by the dominant role of ragweed in that disease. This is in contrast to tree allergy, for which obtaining a pollen extract in concentrations sufficient to produce efficacy should be straightforward; however, the ability to document efficacy is confounded by an inability to identify subjects that demonstrate sensitization to only one or a few trees and by the concomitant presence of innumerable tree-derived pollens, for example, in early spring in the eastern United States. The studies that have shown efficacy in cases of tree allergy involved the treatment of cohorts in locations where a single allergen (eg, birch in Scandinavia or mountain cedar in Texas) presented overwhelming and unique allergic problems. Similarly, the efficacy of IT for grass allergy is well established, reflecting the cross-reactivity of the various northern grass pollens and the relative paucity of confounding allergens during the late spring season.

In contrast to the case of pollens, the ability to show responsiveness to indoor allergens fol- 
lowed a more difficult course and was severely confounded by the absence of a proper characterization of the relevant allergenic proteins. Innumerable patients have received IT for "dust," extracts of which are at best described as "unstandardized" and at worst represent a conglomeration of numerous uncharacterized components, none of which are present at sufficient concentrations to provide any sort of clinical benefit. Only with the recognition of dust mites as the dominant allergen in indoor dust samples did it become possible to develop standardized extracts in concentrations sufficient to mediate clinical benefit. Similarly, the availability of extracts with well-characterized concentrations of the dominant cockroach-derived allergens should make it possible to perform effective IT with these agents. Unfortunately, little additional progress has been made in defining other allergenic proteins likely to be present in indoor dust samples and likely to be producing symptoms in large cohorts of perennial AR subjects. For example, in specific communities, allergens such as those derived from spiders, "miller" moths, and ladybugs may be extremely important sources of allergic symptoms but remain an untapped area of treatment.

Another area in which research has led to the development of efficacious extracts is the treatment of animal allergy. The treatment of subjects with cat allergy is a revealing example of the difficulties inherent in developing useful extracts and the importance of proper investigations. Cats have long been recognized as an important cause of $A R$, and such AR has traditionally been ascribed to cat dander, with little insight in regard to the term "dander." The assumption that the allergen was a peltderived protein led to the development of extracts derived from the skins of carefully cleaned animals. The subsequent recognition that the dominant cat allergen is a protein derived from skin glands led to the recognition of the paradox that the commercially available extracts were virtually devoid of Fel d I and, as such, were worthless as immunomodulating agents. Current standardized extracts, with quantifiable levels of the dominant cat allergen, have proved effective for cat allergy. The treatment of dog allergy has lagged behind that of cat allergy, but the recent characterization of the dominant dog allergens has begun to make available extracts with sufficient concentrations of these allergens to warrant investigations to document their efficacy. Rodents, either as pets or as pests, are an additional major source of animalderived allergens. The rodent glomerulus permits the passage of small proteins, and these urinary proteins are the primary source of allergens. As in the case of cat allergy, only after the recognition that urine and not dander was the source of the dominant allergen has it become possible to develop allergy extracts that contain sufficient concentrations of relevant allergens to plausibly provide efficacy. Continuing research has defined major allergens in other mammals such as horses, cows, and rabbits, and this presumably will lead to the availability of useful extracts.

The one area of inhalant allergy that remains a daunting problem has been the treatment of mould allergies. Although a few studies have shown efficacy of IT in mould-induced allergic disease caused by Alternaria and Cladosporium, the preponderance of studies have not confirmed these results, nor have these results been extended to the innumerable other moulds. In part, this reflects the apparent absence of a single dominant allergen responsible for allergy caused by a given species of mould. Thus, different species (or even strains) of Alternaria have allergens that are sufficiently different that their allergens do not provide crossprotection when administered as IT. In fact, not only do different strains of moulds produce sufficiently different allergenic components to preclude cross-reactive efficacy, but even the same strain can express different allergenic proteins, depending on variations in growth and environmental conditions.

\section{Dose and Duration}

The efficacy of IT is entirely dependent on achieving an optimal therapeutic dose of each allergen. ${ }^{5}$ Low-dose IT (eg, Rinkel therapy) has proved ineffective. It is generally accepted that there is a positive dose-response for most aeroallergen IT. Historically, however, there has been no clear definition as to what constitutes high- or moderate- 
Table 3 Major Allergen Content of US Standardized Extracts*广

\begin{tabular}{lllc}
\hline Allergen Extract & Expressed Potency & Major Allergen & $\begin{array}{c}\text { Mean Content } \\
(\boldsymbol{\mu g} / \mathbf{m L})\end{array}$ \\
\hline Grasses & & & \\
Kentucky blue & $100,000 \mathrm{BAU} / \mathrm{mL}$ & Poa p 5 & 262 \\
Timothy & $100,000 \mathrm{BAU} / \mathrm{mL}$ & Phl p 5 & 743 \\
Orchard & $100,000 \mathrm{BAU} / \mathrm{mL}$ & Dac g 5 & 918 \\
Fescue & $100,000 \mathrm{BAU} / \mathrm{mL}$ & Fes p 5 & 152 \\
Rye & $100,000 \mathrm{BAU} / \mathrm{mL}$ & Lol p 5 & 337 \\
Short ragweed & $1: 10 \mathrm{w} / \mathrm{v}$ & Amb a I & 268 \\
& $\ddagger 1: 20 \mathrm{w} / \mathrm{v}$ & Amb a I & 89 \\
Mixed ragweed & $\$ 1: 10 \mathrm{w} / \mathrm{v}$ & Amb a I & $350-625$ \\
Dermatophagoides & $1: 10 \mathrm{w} / \mathrm{v}$ & Amb a I & 174 \\
pteronyssinus & $10,000 \mathrm{AU} / \mathrm{mL}$ & Der p I & 172 \\
& $\ddagger 10,000 \mathrm{AU} / \mathrm{mL}$ & Der p I & 78 \\
Dermatophagoides farinae & $\$ 30,000 \mathrm{AU} / \mathrm{mL}$ & Der p I & 182 \\
& $10,000 \mathrm{AU} / \mathrm{mL}$ & Der f I & 44 \\
& $\ddagger 10,000 \mathrm{AU} / \mathrm{mL}$ & Der f I & 68 \\
Cat hair & $\ddagger 30,000 \mathrm{AU} / \mathrm{mL}$ & Der f I & 293 \\
& $10,000 \mathrm{BAU} / \mathrm{mL}$ & Fel d I & 40 \\
Dog hair & $\ddagger 10,000 \mathrm{BAU} / \mathrm{mL}$ & Fel d I & 60 \\
Acetone precipitated dog\|l & $10,000 \mathrm{BAU} / \mathrm{mL}$ & Can f I & $40-80$ \\
\hline
\end{tabular}

$\mathrm{AU}=$ allergy units; $\mathrm{BAU}=$ bioequivalent allergy units; $\mathrm{w} / \mathrm{v}=$ weight per volume.

*Sources of extracts: US Food and Drug Administration and extract laboratories.

†Values provided by ALK-Abello, Inc, Wallingford, CT.

Hollister-Stier Laboratories, Spokane, WA.

${ }^{\S}$ Greer Laboratories, Lenoir, NC.

"Hollister-Stier Laboratories, Spokane, WA; now also available in 1:100 with similar Can $\mathrm{f} 1$ values.

dose IT because of a lack of consensus on measurements of potency. Allergen standardization has tremendously advanced our ability to reproducibly administer effective IT extracts. ${ }^{3}$ Standardized extracts are currently available from dust mites, several grasses, short ragweed, and cats and have been evaluated for their major allergen content (Table 3). Other allergen extracts, such as those from dogs, cockroaches, and a few trees and moulds, have not been fully standardized, but at least information regarding the content of relevant allergenic components is available.

With the standardization of allergenic extracts and the availability of information regarding relevant allergens, it became possible to quantify the concentration of allergens used in studies demonstrating the efficacy of IT. Maintenance doses for allergens showing clinical efficacy are summarized in Table 4 . When these data became available, the consistency of the doses showing efficacy was an astounding observation with important clinical significance. Virtually all of the studies that showed efficacy demonstrated this efficacy in a surprisingly narrow range of concentrations - approximately 5 to $24 \mu \mathrm{g}$ per dose of the major allergen. This has two important implications. From an immunologic viewpoint, this suggests that these are the concentrations of antigen required to drive an immune response into tolerance. Of greater relevance to allergists is the implication that this might extend to all allergens and that given information regarding the concentration of major allergens, it should be possible to predict the effective concentration of any extract. Nonstandardized extracts continue to be provided weight per volume or in protein 
Table 4 Recommended Maintenance Doses for Aeroallergen Immunotherapy

\begin{tabular}{|c|c|c|c|}
\hline Allergen & $\begin{array}{c}\text { Dose in } \\
\text { Standardized Units }\end{array}$ & $\begin{array}{c}\text { Dose of } \\
\text { Major Allergen }\end{array}$ & $\begin{array}{c}\text { Maintenance } \\
\text { Concentrate* }(w / v)^{*}\end{array}$ \\
\hline $\begin{array}{l}\text { Dermatophagoides } \\
\text { pteronyssinus }\end{array}$ & $600 \mathrm{AU}$ & $7-12 \mu \mathrm{g}$ Der $\mathrm{p} 1$ & NA \\
\hline $\begin{array}{l}\text { Dermatophagoides } \\
\text { farinae }\end{array}$ & $2,000 \mathrm{AU}$ & $10 \mu \mathrm{g}$ Der $\mathrm{f} 1$ & NA \\
\hline Cat dander & $2,000-3,000 \mathrm{BAU}$ & $11-17 \mu \mathrm{g}$ Fel d 1 & NA \\
\hline Grass & 4,000 BAU & $7-20 \mu \mathrm{g}$ Phi p 5 & NA \\
\hline Short ragweed & NA & $6-24 \mu \mathrm{g} \mathrm{Amb}$ a 1 & $1: 100-1: 30$ \\
\hline $\begin{array}{l}\text { Other pollen } \\
\text { (nonstandardized) }\end{array}$ & NA & ND & $1: 100-1: 30$ \\
\hline Fungi/mould & NA & ND & $1: 100-1: 50$ \\
\hline
\end{tabular}

Adapted from Nelson HS 3 ; Joint Task Force on Practice Parameters ${ }^{5}$; Nelson HS ${ }^{48}$

$\mathrm{AU}=$ allergy units; $\mathrm{BAU}=$ bioequivalent allergy units; $\mathrm{NA}=$ not applicable; $\mathrm{ND}=$ not determined; $\mathrm{w} / \mathrm{v}=$ weight per volume.

*Based on a maintenance injection of $0.5 \mathrm{~mL}$.

nitrogen units. At present, for these nonstandardized allergens, the dose must be based on arbitrary decisions regarding dilution, and this contributes to their limited proven efficacy.

Several other issues pertain to the preparation of extracts for administration. It is important to avoid the inadvertent administration of subtherapeutic doses resulting from dilution caused by mixing multiple allergens. Current guidelines recommend diluting all allergic extracts into the same final volume, regardless of the number of allergens included, and adjusting the volume of diluent in accordance with this number of extracts. In addition, surprisingly little is known at present regarding the stability of these extracts. Loss of extract potency is generally viewed as being accelerated by passage of time, higher temperature, greater dilution, nonglycerine-containing preservatives, allergens with higher protease content, and (in some studies) use of excessive volume for the storage vial. The majority of published studies of concentrated extracts preserved with $50 \%$ glycerin showed the extracts to be stable for at least 12 months. ${ }^{28-33}$ In one study, several allergens remained stable after storage for as long as 36 months at $6^{\circ} \mathrm{C} .{ }^{31}$ However, in contrasting studies, the potency of Der $\mathrm{p} 1$ and Der $\mathrm{p} 2$ fell $50 \%$ at 12 months, ${ }^{32}$ and another study found that Candida lost stability after only 10 weeks. ${ }^{34}$

Several studies have addressed the stability of diluted extracts. Dilutions (1:100) of Russian thistle solubilized in human serum albumin, glycerin, saline, or phosphate buffer maintained potency for 12 months ${ }^{35}$ However, only extracts in glycerin maintained potency at a 1:10,000 dilution. In a different study, temperature had no effect, and at 3 months, diluted extracts that were stored at $4^{\circ} \mathrm{C}$ and those that were brought to room temperature 13 hours a week showed equivalent potency. ${ }^{36}$ At 12 months, both groups had similarly reduced potency. There were no differences in the residual potency between single and mixed allergens except at dilutions of 1:1000, at which there was greater preservation of allergen potency with the three-allergen mix.

A more recent study addressed the influence of dilution and antigen mixing on extract stability. ${ }^{37}$ Potency was preserved at 3 months with single-allergen extracts but was reduced by proteasecontaining extracts; Alternaria had the broadest degradation capacity, followed by cockroach and Cladosporium. Glycerin protected the extracts from the protease-containing allergens. The best preservative was $50 \%$ glycerin, followed by $10 \%$ glycerin and $0.03 \%$ human serum albumin.

In summary, concentrated extracts appear to maintain potency for at least 12 months, and current recommendations state that purchased extracts should therefore be replaced annually. Guidelines argue against the mixing of allergens with high protease activity (such as mould and cockroach allergens) with those of low activity (grass, tree, weed, 
Table 5 Risk Factors for Systemic Reactions to Allergen Immunotherapy

Asthma: poorly controlled or moderate to severe by classification

Administration of immunotherapy in medically unsupervised or unprepared clinical setting

Failure to administer epinephrine

Incorrect injection

Lack of enforcement of the recommended 20- to 30-minute waiting period

Comorbid medical conditions (ie, cardiovascular disease or nonallergic respiratory disease)

Coadministration of pharmacologic therapies: $\beta$-blockers (possibly ACE inhibitors)

Medical noncompliance

$\mathrm{ACE}=$ angiotensin-converting enzyme.

cat, and dog allergens). Glycerinated extracts have the greatest stability and may also act to protect the allergens from proteases.

Current recommendations are that inhalant allergen IT be discontinued after 3 to 5 years. ${ }^{5}$ The most important randomized controlled trial to examine the persistence of improved symptoms after discontinuation of IT was the grass SAR study previously discussed. ${ }^{16}$ This study demonstrated that the IT-induced relief of grass pollen rhinitis symptoms persisted for a minimum of 3 years after discontinuation of IT. No differences were observed between those patients who continued with IT and those who discontinued IT after the completion of 3 years of therapy. Persistence of improved symptoms after discontinuation of dust mite IT has also been shown ${ }^{17}$ although not in a double-blind placebo-controlled trial. Whether IT with other aeroallergens will have the same prolonged protective effects as IT with grass or dust mite allergen has not been determined. Patients will have achieved all of the clinical benefit they can expect from a given extract after 3 to 5 years. Insufficient response to that extract clearly warrants its discontinuation and a reassessment of the diagnosis of AR, the continuing relevance of allergens present in that extract, and the adequacy of dosing of each allergen. Recrudescence of symptoms after IT is discontinued would also warrant a reevaluation of the patient's allergies and suggests that IT may need to be restarted with different agents or with doses that are more appropriate; if allergies to the same allergens remain responsible for the recurrent symptoms, this argues that the repeat IT should be maintained for a longer duration.

\section{Safety Issues}

From local reaction to systemic anaphylaxis and death, the risks from IT require vigilance from physicians, ancillary staff, and the patients themselves. In the United States, severe reactions are rare; estimates range from $<1 \%$ of patients who receive conventional IT to $>36 \%$ of patients in some studies of IT using "rush" regimens involving accelerated buildup of the IT to maintenance doses over a 1 or 2 day period. ${ }^{38,39}$ In a recent review, 41 IT fatalities from 1990 to 2001 were reported, for an average of 3.4 fatal IT reactions per year. ${ }^{40}$ Surprisingly, although incorrect allergy injections were not identified in this study as a cause of death, a survey of allergists' experiences with incorrect allergy injections found that injections given either to the wrong patient or to the correct patient but involving an incorrect dilution or volume were responsible for a systemic reaction rate of approximately $32 \% .{ }^{41}$ Table $\mathrm{V}$ outlines risk factors for systemic reactions to immunotherapy.

Poorly controlled asthma stands as the most significant risk factor for severe systemic reactions to IT (this is also true for all forms of anaphylaxis). Several studies ${ }^{3}$ reported the presence of moderate or severe asthma in the vast majority of reported IT fatalities.$^{38,42}$ More recently, Bernstein and colleagues found that 15 of 17 IT fatality patients had preexisting asthma ${ }^{40} ; 60 \%$ of these patients were reported to have suboptimal control of their asthma symptoms despite appropriate pharmacotherapy. Additionally, 50\% of the asthma fatality patients in this study (for whom data were available) demonstrated a forced expiratory volume in 1 second (FEV1) of $<70 \%$ prior to injection. The 
absence of recent spirometric or even peak flow data for many of these patients emphasizes the importance of obtaining this information on all asthmatic patients prior to administering each IT injection. Symptomatic patients, patients with a recent history of hospitalization or emergency department visits, and patients who have suffered a recent exacerbation of asthma represent the majority of fatal reactors in surveys of the safety of IT. In addition to spirometry or peak flow assessment, preinjection assessments of recent asthma symptoms, rescue inhaler use, nocturnal awakenings, recent health care utilization, and medication compliance are vital for ensuring safe IT for asthmatic patients. Careful selection of patients and the identification of patients with moderate or severe asthma and labile asthma will greatly reduce the risk of fatal systemic reactions to IT.

The potential for severe systemic reactions following IT injections necessitates the administration of IT in a proper clinical setting. The home and other medically unsupervised settings are inappropriate venues for IT; out-of-office administration has been associated with death, likely because of the lack of recognition of systemic reactions and the failure to administer epinephrine in a timely fashion. Guidelines recommend that injections be given at the prescribing allergist's office or at the office of another physician who is trained and medically equipped for the treatment of systemic IT reactions. Delayed administration of epinephrine is consistently reported as a risk factor for IT fatality. In general, epinephrine (1:1000) should be premeasured and instantly available for intramuscular administration should a systemic reaction occur. Physicians who administer IT supplied to them by an allergist must be provided with specific instructions emphasizing the importance of prompt administration of epinephrine and other resuscitative measures to ensure the safety.

Physicians must enforce the recommended 20- to 30-minute waiting period following an IT injection. In general, 20 minutes is considered sufficient for patients with AR alone whereas asthmatic patients should remain at least 30 minutes after an IT injection. However, up to $38 \%$ of systemic reactions occur after 30 minutes and thus outside the clinician's office. Current recommenda- tions advise a prolonged postinjection waiting period for patients with a history of systemic reactions after the half-hour waiting period. ${ }^{5}$ Although no more-specific recommendations exist, these observations argue for the appropriateness of more-prolonged waiting periods for patients with more than mild asthma or with a history of a previous systemic reaction. In addition, it has been suggested that any patient with a history of a previous systemic reaction or perhaps even all asthmatic patients receiving IT should be prescribed epinephrine and should receive appropriate training in its indications and administration.

Patient-specific IT vials and administration forms may reduce the risk of incorrect IT injections. As $32 \%$ of incorrect IT injections potentially lead to systemic reactions, specific steps must be taken at each patient encounter to ensure safety. One suggested approach involves a "one patient, one nurse, one injection" safety measure. One nurse and the patient review three identifiers, including first and last names on the patientspecific IT vial and administration form, as well as the patient's date of birth or medical record number. An additional recommendation is to use standardized color-coded IT vials according to strength (from most dilute to maintenance strength). Finally, clinic physicians should be informed immediately should there be any deviation from an appropriate injection dose.

On initial and follow-up evaluations of patients with AR or allergic asthma, close attention to comorbid medical conditions and coadministration of pharmacologic therapies are essential for safe IT. Patients may have heart disease or hypertension requiring medications such as $\beta$-blockers or angiotensin-converting enzyme inhibitors, both of which have been implicated in fatal reactions to IT. ${ }^{5,38,40}$ Advanced age, nonallergic respiratory disease, or risk factors for cardiovascular disease such as hypertension, elevated cholesterol, or diabetes should be weighed in risk-benefit analyses because administration of epinephrine for systemic reactions may induce significant cardiovascular side effects. These patients may also have a reduced ability to survive a systemic reaction. Although not addressed here, Hymenoptera IT benefits may outweigh risk in patients taking 
$\beta$-blockers insofar as these patients are unlikely to be able to completely avoid stings in the future and are at less risk from the complications of a systemic reaction in a physician's office than they would be in the field after being stung.

\section{Future Immunotherapy Strategies}

Future strategies for IT are aimed at delivering increased efficacy with less risk of IgE-mediated systemic side effects. Recognition of the importance of T-cell-dependent mechanisms in immunomodulation after IT has led to studies to develop reagents that interact with $\mathrm{T}$ lymphocytes without engaging mast cell/basophil-bound IgE. Denatured proteins such as polymerized extracts or, more recently, short peptide fragments derived from genetically-engineered allergens have been successfully used in IT studies. These peptides retain the ability to be recognized and processed by antigen-presenting cells, to be presented to $\mathrm{T}$ helper lymphocytes, and to drive $\mathrm{T}$ cells into an anergic or tolerant state. Because these short peptides lack the complex structure of their parent compounds, they cannot be recognized by mast cell/basophil-bound IgE and are generally not associated with risk for immediate-phase anaphylactic responses. Studies of peptides in patients sensitized to cats ${ }^{43,44}$ and to bee venom ${ }^{45}$ have demonstrated reductions in the proliferation of $\mathrm{CD} 4^{+} \mathrm{T}$ cells, diminished $\mathrm{Th} 1$ and $\mathrm{Th} 2$ cytokine production, and significant increases in IL-10. However, these compounds have been frustrated by their tendency initially to activate allergenspecific Th2-like cells and thereby produce delayed but often severe allergic reactions, including exacerbations of asthma. ${ }^{46}$ More recent studies have used allergenic peptides coupled to synthetic bacterial deoxyribonucleic acid (DNA) sequences. ${ }^{47}$ These are based on the concept that engagement of these immunostimulatory DNA sequences to their specific toll-like 9 (TL9) receptors will be a potent signal to drive the ensuing immune responses toward Th1 immunity. Initial studies were extremely promising in regard to efficacy, safety, and long-term immunomodulation with these agents. Interestingly, the absence of adverse effects associated with the Th1-like responses appears to be related to a concomitant induction of regulatory immune responses.

A final and potentially more immediately available possibility involves the concurrent administration of humanized anti-IgE (omalizumab) with standard IT. The concept is that the removal of IgE should dramatically reduce the risk of anaphylactic reactions and thereby may allow a more rapid induction of tolerance, the implementation of higher doses of allergen, or the administration of IT to patients who are considered to have contraindications to IT. For example, it may become easier to administer IT to patients with moderate to severe asthma or with a previous history of systemic reactions. Recent studies support such an approach and raise the exciting potential of a role for IT in patients for whom immunomodulating therapies might provide critically important clinical benefits.

\section{References}

1. Canadian Society of Allergy and Clinical Immunology. Guidelines for the use of allergen immunotherapy. Can Med Assoc J 1995; 152:1413-9.

2. WHO position paper. Allergen immunotherapy: therapeutic vaccines for allergic diseases. Allergy 1998;47:698-704.

3. Nelson HS. The use of standardized extracts in allergen immunotherapy. J Allergy Clin Immunol 2000;106(1 Pt 1):41-5.

4. Lockey RF. "ARIA": global guidelines and new forms of allergen immunotherapy. J Allergy Clin Immunol 2001;108:497-9.

5. Joint Task Force on Practice Parameters. Allergen immunotherapy: a practice parameter. American Academy of Allergy, Asthma and Immunology. American College of Allergy, Asthma and Immunology. Ann Allergy Asthma Immunol 2003;90(1 Suppl 1):1-40.

6. Jutel M, Pichler WJ, Skrbic D, et al. Bee venom immunotherapy results in decrease of IL-4 and IL-5 and increase of IFN-gamma secretion in specific allergen-stimulated $\mathrm{T}$ cell cultures. J Immunol 1995;154:4187-94. 
7. Hansen G, Berry G, DeKruyff RH, Umetsu DT. Allergen-specific Th1 cells fail to counterbalance Th2 cell-induced airway hyperreactivity but cause severe airway inflammation. J Clin Invest 1999;103:175-83.

8. Akdis CA, Blesken T, Akdis M, et al. Role of interleukin 10 in specific immunotherapy. J Clin Invest 1998;102:98-106.

9. Jutel M, Akdis M, Budak F, et al. IL-10 and TGF-beta cooperate in the regulatory $\mathrm{T}$ cell response to mucosal allergens in normal immunity and specific immunotherapy. Eur $\mathrm{J}$ Immunol 2003;33:1205-14.

10. Francis JN, Till SJ, Durham SR. Induction of IL-10+CD4+CD25+ $\mathrm{T}$ cells by grass pollen immunotherapy. J Allergy Clin Immunol 2003;111:1255-61.

11. Lowell FC, Franklin W. A double-blind study of the effectiveness and specificity of injection therapy in ragweed hay fever. N Engl J Med 1965;273:675-9.

12. Ohashi Y, Nakai Y, Tanaka A, et al. Serologic study of the working mechanisms of immunotherapy for children with perennial allergic rhinitis. Arch Otolaryngol Head Neck Surg 1998;124:1337-46.

13. Ross RN, Nelson HS, Finegold I. Effectiveness of specific immunotherapy in the treatment of allergic rhinitis: an analysis of randomized, prospective, single- or double-blind, placebocontrolled studies. Clin Ther 2000;22:342-50.

14. Cantani A, Micera M. Is specific immunotherapy safe and effective in children? Eur Rev Med Pharmacol Sci 2000;4:139-43.

15. Pichler CE, Helbling A, Pichler WJ. Three years of specific immunotherapy with housedust-mite extracts in patients with rhinitis and asthma: significant improvement of allergenspecific parameters and of nonspecific bronchial hyperreactivity. Allergy 2001;56:301-6.

16. Durham SR, Walker SM, Varga EM, et al. Long-term clinical efficacy of grass-pollen immunotherapy. N Engl J Med 1999;341: 468-75.

17. Des Roches A, Paradis L, Knani J, et al. Immunotherapy with a standardized Dermatophagoides pteronyssinus extract. V. Duration of the efficacy of immunotherapy after its cessation. Allergy 1996;51:430-3.
18. Des Roches A, Paradis L, Menardo JL, et al. Immunotherapy with a standardized Dermatophagoides pteronyssinus extract. VI. Specific immunotherapy prevents the onset of new sensitizations in children. J Allergy Clin Immunol 1997;99:450-3.

19. Cools M, Van Bever HP, Weyler JJ, Stevens WJ. Long-term effects of specific immunotherapy, administered during childhood, in asthmatic patients allergic to either house-dust mite or to both house-dust mite and grass pollen. Allergy 2000;55:69-73.

20. Moller C, Dreborg S, Ferdousi HA, et al. Pollen immunotherapy reduces the development of asthma in children with seasonal rhinoconjunctivitis (the PAT study). J Allergy Clin Immunol 2002;109:251-6.

21. Johnstone DE, Dutton A. The value of hyposensitization therapy for bronchial asthma in children-a 14-year study. Pediatrics 1968;42:793-802.

22. Adkinson NF Jr, Eggleston PA, Eney D, et al. A controlled trial of immunotherapy for asthma in allergic children. N Engl J Med 1997;336: 324-31.

23. Cantani A, Arcese G, Lucenti P, et al. A threeyear prospective study of specific immunotherapy to inhalant allergens: evidence of safety and efficacy in 300 children with allergic asthma. J Investig Allergol Clin Immunol 1997;7:90-7.

24. Hedlin G, Wille S, Browaldh L, et al. Immunotherapy in children with allergic asthma: effect on bronchial hyperreactivity and pharmacotherapy. J Allergy Clin Immunol 1999;103:609-14.

25. Portnoy JM. Immunotherapy for asthma: unfavorable studies. Ann Allergy Asthma Immunol 2001;87(1 Suppl 1):28-32.

26. Abramson MJ, Puy RM, Weiner JM. Is allergen immunotherapy effective in asthma? A metaanalysis of randomized controlled trials. Am J Respir Crit Care Med 1995;151:969-74.

27. Ross RN, Nelson HS, Finegold I. Effectiveness of specific immunotherapy in the treatment of asthma: a meta-analysis of prospective, randomized, double-blind, placebo-controlled studies. Clin Ther 2000;22:329-41.

28. Center JG, Shuller N, Zeleznick LD. Stability of antigen $\mathrm{E}$ in commercially prepared ragweed 
pollen extracts. Dev Biol Stand 1975;29: 114-22.

29. Ayuso R, Rubio M, Herrera T, et al. Stability of Lolium perenne extract. Ann Allergy 1984;53: 426-31.

30. Vijay HM, Young NM, Bernstein IL. Studies on Alternaria allergens. VI. Stability of the allergen components of Alternaria tenuis extracts under a variety of storage conditions. Int Arch Allergy Appl Immunol 1987;83:325-8.

31. Niemeijer NR, Kauffman HF, van Hove W, et al. Effect of dilution, temperature, and preservatives on the long-term stability of standardized inhalant allergen extracts. Ann Allergy Asthma Immunol 1996;76:535-40.

32. Liu T, Lin Y. The epitope stability of group 1 and group 2 allergens in mite extracts. Ann Allergy Asthma Immunol 1998;80:177-83.

33. Soldatova LN, Paupore EJ, Burk SH, et al. The stability of house dust mite allergens in glycerinated extracts. J Allergy Clin Immunol 2000;105:482-8.

34. Savolainen J, Broberg A. Crossreacting IgE antibodies to Pityrosporum ovale and Candida albicans in atopic children. Clin Exp Allergy 1992;22:469-74.

35. Nelson HS. The effect of preservatives and dilution on the deterioration of Russian thistle (Salsola pestifer), a pollen extract. J Allergy Clin Immunol 1979;63:417-25.

36. Nelson HS. Effect of preservatives and conditions of storage on the potency of allergy extracts. J Allergy Clin Immunol 1981; 67:64-9.

37. Nelson HS, Ikle D, Buchmeier A. Studies of allergen extract stability: the effects of dilution and mixing. J Allergy Clin Immunol 1996;98:382-8.

38. Lockey RF, Nicoara-Kasti GL, Theodoropoulos DS, Bukantz SC. Systemic reactions and fatalities associated with allergen immunotherapy. Ann Allergy Asthma Immunol 2001;87(1 Suppl 1):47-55.
39. Malling HJ. Allergen-specific immunotherapy in allergic rhinitis. Curr Opin Allergy Clin Immunol 2001;1:43-6.

40. Bernstein DI, Wanner M, Borish L, Liss GM. Twelve-year survey of fatal reactions to allergen injections and skin testing: 1990-2001. J Allergy Clin Immunol 2004;113:1129-36.

41. Aaronson DW, Gandhi TK. Incorrect allergy injections: allergists' experiences and recommendations for prevention. J Allergy Clin Immunol 2004;113:1117-21.

42. Reid MJ, Lockey RF, Turkeltaub PC, PlattsMills TA. Survey of fatalities from skin testing and immunotherapy 1985-1989. J Allergy Clin Immunol 1993;92:6-15.

43. Haselden BM, Kay AB, Larche M. Peptidemediated immune responses in specific immunotherapy. Int Arch Allergy Immunol 2000;122:229-37.

44. Oldfield WL, Kay AB, Larche M. Allergenderived $T$ cell peptide-induced late asthmatic reactions precede the induction of antigen-specific hyporesponsiveness in atopic allergic asthmatic subjects. J Immunol 2001;167: 1734-9.

45. Muller U, Akdis CA, Fricker M, et al. Successful immunotherapy with T-cell epitope peptides of bee venom phospholipase A2 induces specific T-cell anergy in patients allergic to bee venom. J Allergy Clin Immunol 1998;101:747-54.

46. Larche M, Haselden BM, Oldfield WL, et al. Mechanisms of $\mathrm{T}$ cell peptide epitope-dependent late asthmatic reactions. Int Arch Allergy Immunol 2001;124:272-5.

47. Tighe H, Takabayashi K, Schwartz D, et al. Conjugation of immunostimulatory DNA to the short ragweed allergen amb a 1 enhances its immunogenicity and reduces its allergenicity. $\mathbf{J}$ Allergy Clin Immunol 2000;106:124-34.

48. Nelson HS. Immunotherapy for inhalant allergens. In: Adkinson NF Jr, Yunginger JW, Busse WW, et al, editors. Middleton's allergy: principles and practice. 6th ed. Philadelphia: C. V. Mosby; 2003:1455-74. 